\title{
PRESENCIA DE Enterobacteriaceae y Escherichia coli MULTIRRESISTENTE A ANTIMICROBIANOS EN CARNE ADQUIRIDA EN MERCADOS TRADICIONALES EN LIMA
}

\author{
Lidia Ruiz-Roldán ${ }^{1, a}$, Sandra Martínez-Puchol1,a ${ }^{1,}$ Cláudia Gomes ${ }^{1, b}$, Noemí Palma ${ }^{1, a}$, Maribel Riveros ${ }^{2, c}$, \\ Karen Ocampo ${ }^{2, c}$, David Durand ${ }^{2, c}$, Theresa J. Ochoa ${ }^{2,3, d}$, Joaquim Ruiz ${ }^{1, b}$, Maria J. Pons ${ }^{4, b}$
}

\begin{abstract}
RESUMEN
Objetivos. El objetivo del presente estudio fue describir la presencia de Enterobacteriaceae en muestras de carne recolectadas en mercados tradicionales de Lima y establecer los niveles de resistencia a antimicrobianos y la presencia de betalactamasas de espectro extendido (BLEE) y AmpC en Escherichia coli. Materiales y métodos. Se recolectaron un total de 138 muestras de carne, 64 (46,4 \%) de pollo, 44 (31,9\%) de carne de res y 30 (21,7\%) de carne de cerdo. Las bacterias aisladas pertenecieron a 17 géneros diferentes, y específicamente 14 fueron clasificados como Enterobacteriaceae. Se analizó la sensibilidad frente a diez agentes antimicrobianos mediante el método de difusión de disco Kirby-Bauer, se determinó la presencia de BLEE y AmpC mediante las pruebas de doble disco y de inducción de imipenem-ceftazidima, respectivamente. Resultados. Los niveles de resistencia a los antimicrobianos fueron altos frente a trimetoprima-sulfametoxazol, ampicilina, tetraciclina, ácido nalidíxico, ciprofloxacino y cloranfenicol. Existen diferencias significativas en los niveles de resistencia a los antibióticos según el tipo de carne (pollo, carne de res y cerdo) $(p<0,05)$. Los niveles de resistencia a múltiples antimicrobianos (MDR) fueron particularmente altos en pollo y cerdo ( $98,2 \%$ y $86,4 \%$, respectivamente). Además, la presencia de BLEE en Escherichia coli aisladas de carne de pollo fue del $59,4 \%$. Conclusiones. Los niveles de resistencia a los antimicrobianos fueron altos frente a los antibióticos usados frecuentemente en humanos, se destaca el pollo y la res como potenciales reservorios de Escherichia coli productoras de BLEE y pAmpC, respectivamente.
\end{abstract}

Palabras clave: Carne; Alimentos; Resistencia a betalactamasas; Resistencia a multidrogas; Escherichia coli; Perú; América Latina (fuente: DeCS BIREME).

\section{PRESENCE OF MULTIDRUG RESISTANT Enterobacteriaceae and Escherichia coli IN MEAT PURCHASED IN TRADITIONAL MARKETS OF LIMA}

\begin{abstract}
Objective. The objective of this study was to describe the presence of Enterobacteriaceae in meat samples collected in traditional markets of Lima and to establish the levels of antimicrobial resistance and the presence of extended spectrum beta-lactamases (BLEE) and AmpC in Escherichia coli. Materials and Methods. A total of 138 meat samples, 64 (46.4\%) chicken, $44(31.9 \%)$ beef and $30(21.7 \%)$ pork were collected. The isolated bacteria belonged to 17 different genera and, specifically, 14 were classified as Enterobacteriaceae. Sensitivity to ten antimicrobial agents was analyzed using the Kirby-Bauer disc diffusion method, BLEE and AmpC were determined by double disc and imipenem-ceftazidime induction tests, respectively. Results. Antimicrobial resistance levels were high against trimethoprim-sulfamethoxazole, ampicillin, tetracycline, nalidixic acid, ciprofloxacin and chloramphenicol. There are significant differences in antibiotic resistance levels depending on the type of meat (chicken, beef and pork) $(p<0.05)$. Multiple drug resistance (MDR) levels were particularly high in chicken and pork $(98.2 \%$ and $86.4 \%$, respectively). In addition, the presence of BLEE in Escherichia coli isolated from chicken meat was 59.4\%. Conclusions. Multiple drug resistance levels were high compared to antibiotics frequently used in humans; chicken and beef are highlighted as potential reservoirs of BLEE and pAmpC-producing Escherichia coli, respectively.
\end{abstract}

Keywords: Meat; Food; Resistance to beta-lactamases; Multidrug resistance; Escherichia coli; Perú; Latin America (source: MeSH NLM).

\footnotetext{
ISGlobal, Hospital Clínic - Universitat de Barcelona, Barcelona, Spain.

Universidad Peruana Cayetano Heredia, Instituto de Medicina Tropical Alexander Von Humboldt. Lima, Perú.

Center for Infectious Disease, University of Texas School of Public Health. Houston, USA.

Universidad Científica del Sur, Lima, Perú.

Magister en Biología; ${ }^{\mathrm{b}} \mathrm{PhD}$ en Biología; ${ }^{\mathrm{c}}$ licenciado en Biología; ${ }^{\mathrm{d}}$ medico infectóloga

Recibido: 15/06/2018 Aprobado: 12/09/2018 En línea: 28/09/2018
}

Citar como: Ruiz-Roldán L, Martínez-Puchol S, Gomes C, Palma N, Riveros M, Ocampo K, et al. Presencia de Enterobacteriaceae y Escherichia coli multirresistente a antimicrobianos en carne adquirida en mercados tradicionales en Lima. Rev Peru Med Exp Salud Publica. 2018;35(3):425-32. doi:10.17843/ rpmesp.2018.353.3737. 


\section{INTRODUCCIÓN}

Una de las vías de entrada más importantes de los microorganismos al ser humano es a través de la cadena alimentaria. De los microorganismos ingeridos, son los patógenos los que producen enfermedades transmitidas por alimentos ${ }^{(1)}$. En todo el mundo, el consumo de alimentos contaminados ocasiona la muerte de dos millones de personas al año (2).

Diferentes miembros de la familia Enterobacteriaceae, incluidas Salmonella spp., Shigella spp. y los patotipos diarreagénicos de Escherichia coli (E.coli) son considerados microorganismos patógenos transmitidos por alimentos ${ }^{(1-3)}$. Las enterobacteriáceas, aunque se reportan en diferentes tipos de alimentos, se encuentran principalmente en productos derivados de la carne, debido a que forman parte de su microbiota intestinal (3). Del mismo modo, la presencia de Salmonella spp. está vinculada frecuentemente al consumo de pollo, huevos o sus productos derivados ${ }^{(3)}$, mientras que las infecciones por $E$. coli productoras de Shiga toxina (STEC) se relacionan con el consumo de carne de vacuno ${ }^{(4)}$.

La mayoría de los microorganismos que se aíslan de muestras de alimentos no son patógenos, teniendo a la $E$. coli como el ejemplo mejor caracterizado. Estos microorganismos son de gran relevancia por formar parte de la microbiota intestinal humana, actuando como reservorios de genes de resistencia a antibióticos ${ }^{(5)}$. Estos genes pueden transferirse a otros microorganismos directamente (por ejemplo, mediante conjugación) o indirectamente (por ejemplo, mediante transformación después de la lisis bacteriana y la liberación de ADN en el entorno intestinal), pudiendo llegar a microorganismos patógenos durante el curso de infecciones o colonizaciones transitorias ${ }^{(6)}$.

La resistencia a los antimicrobianos es un problema de salud que sigue incrementándose a nivel mundial. En la mayoría de los países de bajos y medianos ingresos el problema está subestimado, principalmente por la escasez de estudios en estas regiones. Asimismo, los datos disponibles actualmente han mostrado un escenario preocupante, con altos niveles de resistencia a los antimicrobianos usados comúnmente ${ }^{(7)}$. La presencia de betalactamasas de espectro extendido (BLEE) es uno de los mecanismos de resistencia a antimicrobianos más importantes ${ }^{(7)}$. Estas enzimas suelen estar codificadas en las mismas estructuras genéticas que otros mecanismos de resistencia, lo que da lugar a fenotipos de multirresistencia a los antibióticos.

Otro mecanismo de resistencia (a las cefalosporinas) es el llamado AmpC mediado por plásmidos (pAmpC) que, a pesar de ser poco frecuente, también ha sido reportado en Perú, específicamente en muestras recolectadas en hospitales ${ }^{(8)}$.

\section{MENSAJES CLAVE}

Motivación para realizar el estudio. Las bacterias presentes en carne, puede causar infecciones; además, pueden ser una importante vía de transmisión de la resistencia a los antibióticos.

Principales hallazgos. Los niveles de resistencia fueron altos sobre todo en aquellos antibióticos usados frecuentemente en humanos. La carne de pollo presentó los niveles de resistencia más elevados; además de E.coli productoras de betalactamasas de espectro extendido (BLEE).

Implicancias. La presencia de bacterias multirresistentes y productoras de BLEE en la carne de expendio en los mercados de Lima es un importante problema de salud, esto podría deberse al uso indiscriminado de antibióticos en la producción cárnica.

En Perú se han reportado niveles extremadamente altos de resistencia a los antibióticos, siendo de especial preocupación los ocasionados por microorganismos patógenos productores de BLEE ${ }^{(7)}$.

No se dispone de datos sobre la presencia de bacterias multirresistentes en productos cárnicos de los mercados tradicionales de Lima. Este estudio tiene como objetivo determinar la presencia de Enterobacteriaceae en muestras de diferentes tipos de carne (vacuno, cerdo y pollo) así como sus niveles de sensibilidad a antimicrobianos, en especial la presencia de BLEE y pAmpC.

\section{MATERIALES Y MÉTODOS}

\section{DISEÑO, MUESTRA Y PLAN DE MUESTREO}

Se realizó un estudio de diseño descriptivo, transversal y prospectivo. Se recolectaron en total 138 muestras de carne, de las cuales 30 corresponden a cerdo (chuleta), 44 a vacuno (lomo) y 64 a pollo (pierna), de mercados tradicionales del norte (Comas, San Martín), centro (La Victoria, Cercado de Lima) y sur (Villa El Salvador) de Lima. Las muestras se recolectaron de forma aleatoria.

\section{PROCESAMIENTO DE LAS MUESTRAS}

Las muestras se transportaron en bolsas estériles a $4{ }^{\circ} \mathrm{C}$ y se procesaron inmediatamente después de llegar al Laboratorio de Enfermedades Entéricas, Nutrición y Resistencia Antimicrobiana de la Universidad Peruana Cayetano Heredia, en un máximo de $4 \mathrm{~h}$ desde el momento de la compra. Luego, se extrajo $2,5 \mathrm{~g}$ de cada muestra, se enriqueció en caldos de Luria Bertani y de selenita durante una noche. Posteriormente, se sembró $500 \mu$ l en diferentes medios: agar Xylose Lisina Deoxicolato, agar SalmonellaShigella, agar Mc Conkey y agar Hektoen ${ }^{(9)}$. Cada muestra se procesó de acuerdo con las normas internacionales ISO 21567: 2004 para Shigella, e ISO 6579 para Salmonella ${ }^{(10)}$.

\section{IDENTIFICACIÓN DE LAS BACTERIAS}

Los aislados bacterianos se identificaron por su crecimiento en los medios de cultivo, por su morfología en las colonias 
y mediante técnicas bioquímicas convencionales ${ }^{(11)}$ y el kit de identificación de bacterias API® (Biomerieux, Marcyl'Etoile, Francia). Las cepas aisladas identificadas como $E$. coli, se confirmaron mediante la amplificación del gen uid $A$, mientras que las identificadas como Shigella spp. o Salmonella spp. fueron confirmadas por la amplificación de los genes ipaH y invA, respectivamente ${ }^{(12,13)}$. El resto de los microorganismos, así como los que presentaron discrepancias entre los procedimientos bioquímicos y por reacción en cadena de polimerasa (PCR) se identificaron mediante la amplificación y posterior secuenciación de un fragmento del gen $16 S$ rRNA ${ }^{(14)}$. Se consideró sólo un aislamiento, cuando la misma especie se obtuvo más de una vez de la misma muestra.

\section{DETECCIÓN DE E. coli DIARREOGÉNICAS}

El carácter diarreogénico de las cepas de $E$. coli (E. coli enterotoxigénica, ETEC; E. coli enteropatogénica, EPEC; $E$. coli productora de toxina Shiga, STEC; E. coli enteroinvasiva, EIEC; E. coli difusamente adherente, DAEC) fueron confirmados por la técnica de PCR ${ }^{(15)}$

\section{RESISTENCIA ANTIMICROBIANA}

Se determinó la sensibilidad antimicrobiana frente a diez agentes antibacterianos, mediante el método de difusión en disco, siguiendo las directrices de la guía CLSI (Clinical and Laboratory Standards Institute) ${ }^{(9)}$. Estos agentes incluyeron: ampicilina $10 \mu \mathrm{g} / \mathrm{ml}$, amoxicilina más ácido clavulánico 20/10 $\mu \mathrm{g} / \mathrm{ml}$, imipenem $10 \mu \mathrm{g} / \mathrm{ml}$, cloranfenicol $30 \mu \mathrm{g} / \mathrm{ml}$, ácido nalidíxico $30 \mu \mathrm{g} / \mathrm{ml}$, ciprofloxacina $5 \mu \mathrm{g} / \mathrm{ml}$, nitrofurantoína $300 \mu \mathrm{g} / \mathrm{ml}$, cotrimoxazol 1,25/23,75 $\mu \mathrm{g} / \mathrm{ml}$ y tetraciclina 30 $\mu \mathrm{g} / \mathrm{ml}$. Además, para furazolidona $100 \mu \mathrm{g} / \mathrm{ml}$ y azitromicina $15 \mu \mathrm{g} / \mathrm{ml}$. No existe un punto de corte específico en la guía CLSI para la furazolidona, por lo que se utilizó el punto de corte de la nitrofurantoína, y en el caso de la azitromicina se uso el punto de corte propuesto por Pons et al. ${ }^{(16)}$.

Se seleccionó un grupo representativo de cepas de $E$. coli de cada perfil de resistencia para su inclusión en el presente estudio. Se determinó, además, la sensibilidad antibiótica a cefoxitina, cefotaxima, ceftazidima y gentamicina. Seguidamente, se evaluó la presencia fenotípica de BLEE y pAmpC mediante la prueba de doble disco y la prueba de inducción de imipenem-ceftazidima, respectivamente. Se utilizó la cepa E. coli ATCC 25922 como control en los antibiogramas. Se consideró como cepas multidrogorresistentes (MDR) a aquellas que mostraron resistencia, al menos a tres o más familias o grupo de antimicrobianos, no relacionados entre ellos.

\section{ANÁLISIS ESTADÍSTICO}

Las tasas de resistencia antibacteriana en diferentes especies de animales se compararon mediante la prueba de Fisher y Chi cuadrado, según corresponda. Las diferencias obtenidas con un valor de $p \leq 0,05$ se consideraron significativas. Se utilizó el software Stata 13.0 (Stata Corp., College Station, TX). Las frecuencias y porcentajes de la resistencia a los antibióticos se presentaron en las muestras de carne analizadas y solo frecuencias en las bacterias aisladas.

\section{CONSIDERACIONES ÉTICAS}

El proyecto fue aprobado por el Comité de Ética de la Universidad Peruana Cayetano Heredia. En todo momento se mantuvo el anonimato de los puestos de expendio y de los vendedores que proporcionaron las muestras de estudio.

\section{RESULTADOS}

\section{AISLAMIENTO E IDENTIFICACIÓN DE MICROORGANISMOS EN LAS MUESTRAS}

Se analizaron un total de 138 muestras; 30 de carne de cerdo, 44 de carne de res y 64 de pollo. Se identificaron 830 microorganismos pertenecientes a 17 géneros bacterianos, $14(82,4 \%)$ fueron de la familia Enterobacteriaceae (801 aislamientos), los tres (17,6\%) restantes fueron de las familias Aeromonadaceae, Moraxellaceae (solo aisladas de muestras de carne de res) y Pseudomonaceae.

Entre las especies identificadas, las más comunes fueron: Escherichia coli (407, $49 \%$ de los aislados), Providencia spp. (112, 13,5 \%), Proteus spp. (84, 10,1\%), Citrobacterspp. (59, 7,1\%) y Enterobacterspp. (48, 5,8 \%). No se encontraron diferencias en el número de familias de microorganismos dentro de las muestras, se recuperaron 15 géneros diferentes en cada tipo de muestra específica. En general, los promedios de diferentes géneros por tipo de muestra fueron de 2,7 en el caso de la carne de cerdo y vacuno, y 3,2 en el caso de pollo. El género descrito con más frecuencia fue Escherichia spp. (todos identificados como E. coli), seguidos de Providencia spp., Proteus spp. (57 muestras, 41,3 \%), Citrobacter spp. y Enterobacter spp. El género bacteriano más frecuente en las muestras de cerdo fue Escherichia (24 muestras, $80 \%$ ), seguido de Providencia spp. y Enterobacter spp. (cada uno en 14 muestras, 43,3\%). En las muestras de pollo, el género más frecuente fue Escherichia (61 muestras, 95,3\%), seguido de Proteus spp. (37 muestras, 57,8 \%) y Providencia spp. (35 muestras, 54,7 \%). En las muestras de carne de vacuno, se observó la presencia de Escherichia (31 muestras, 70,4 \%), Citrobacter spp. (20 muestras, 45,5\%) y Providencia spp. (15 muestras, 34,1 \%) (Tabla 1).

Asimismo, se encontró diferencias significativas en las frecuencias de los géneros según los tipos de muestra; así, Salmonella spp., Proteus spp. y Escherichia spp. fueron más frecuentes en las muestras de pollo que en las muestras de cerdo ( $p<0,05$; en los tres casos) o de carne de vacuno ( $p<0,05$; en los tres casos). Además, Providencia 
Tabla 1. Especies bacterianas identificadas en muestras de carne procedentes de mercados tradicionales de Lima

\begin{tabular}{|c|c|c|c|c|c|c|c|c|c|}
\hline \multirow{2}{*}{\multicolumn{2}{|c|}{ Microorganismos (830) }} & \multicolumn{8}{|c|}{ Muestras de carne } \\
\hline & & \multicolumn{2}{|c|}{ Total (138) } & \multicolumn{2}{|c|}{ Pollo (64) } & \multicolumn{2}{|c|}{ Vacuno (44) } & \multicolumn{2}{|c|}{ Cerdo (30) } \\
\hline Género y especie* & $\mathbf{n}^{\dagger}$ & $\mathbf{n} \neq$ & $\%$ & $\mathrm{n}$ & $\%$ & $\mathbf{n}$ & $\%$ & $\mathbf{n}$ & $\%$ \\
\hline \multicolumn{10}{|c|}{ Enterobacteriaceae (801) } \\
\hline Citrobacter & 59 & 43 & 31,1 & 14 & 21,9 & 20 & 45,5 & 9 & 30,0 \\
\hline C. freundii & 50 & 38 & 27,5 & 12 & 18,7 & 18 & 40,9 & 8 & 26,7 \\
\hline spp & 9 & 5 & 2,8 & 2 & 3,1 & 2 & 4,5 & 1 & 3,3 \\
\hline Enterobacter & 48 & 43 & 31,1 & 16 & 25,0 & 14 & 31,8 & 13 & 43,3 \\
\hline E. cloacae & 14 & 15 & 10,9 & 7 & 10,9 & 4 & 9,1 & 4 & 6,3 \\
\hline spp & 34 & 26 & 18,8 & 9 & 14,1 & 10 & 22,7 & 7 & 23,3 \\
\hline Escherichia & 407 & 116 & 84,1 & 61 & 95,3 & 31 & 70,4 & 24 & 80,0 \\
\hline E. coli & 407 & 116 & 84,1 & 61 & 95,3 & 31 & 70,4 & 24 & 80,0 \\
\hline Hafnia & 13 & 13 & 9,4 & 6 & 9,4 & 5 & 11,4 & 2 & 6,6 \\
\hline H. alvei & 13 & 13 & 9,4 & 6 & 9,4 & 5 & 11,4 & 2 & 6,6 \\
\hline Klebsiella & 18 & 18 & 13,0 & 6 & 9,4 & 7 & 15,9 & 5 & 16,7 \\
\hline K. oxytoca & 12 & 12 & 8,7 & 3 & 4,7 & 5 & 11,4 & 4 & 13,3 \\
\hline K. pneumoniae & 6 & 6 & 4,3 & 3 & 4,7 & 2 & 4,5 & 1 & 3,3 \\
\hline Moellerella & 1 & 1 & 0,7 & 0 & 0,0 & 0 & 0,0 & 0 & 0,0 \\
\hline Morganella & 10 & 9 & 6,5 & 5 & 7,8 & 2 & 4,5 & 2 & 6,6 \\
\hline Obesumbacterium & 1 & 1 & 0,7 & 0 & 0,0 & 0 & 0,0 & 0 & 0,0 \\
\hline Pantoea & 5 & 5 & 2,8 & 0 & 0,0 & 0 & 0,0 & 0 & 0,0 \\
\hline P. agglomerans & 2 & 2 & 1,4 & 0 & 0,0 & 1 & 2,3 & 1 & 3,3 \\
\hline spp & 3 & 3 & 2,2 & 2 & 3,1 & 1 & 2,3 & 0 & 0,0 \\
\hline Proteus & 84 & 57 & 41,3 & 37 & 57,8 & 11 & 25,0 & 9 & 30,0 \\
\hline P. mirabilis & 74 & 48 & 34,8 & 35 & 54,7 & 7 & 15,9 & 6 & 20,0 \\
\hline spp & 10 & 9 & 6,5 & 2 & 3,1 & 4 & 9,1 & 3 & 10,0 \\
\hline Providencia & 112 & 63 & 45,7 & 35 & 54,7 & 15 & 34,1 & 13 & 43,3 \\
\hline P. alcalifaciens & 82 & 43 & 31,1 & 22 & 34,4 & 11 & 25,0 & 10 & 36,7 \\
\hline spp & 30 & 20 & 14,5 & 13 & 20,3 & 4 & 9,1 & 3 & 10,0 \\
\hline Raoultella & 16 & 12 & 8,7 & 3 & 4,7 & 6 & 13,6 & 3 & 10,0 \\
\hline R. ornithinolytica & 15 & 11 & 7,8 & 3 & 4,7 & 5 & 11,4 & 3 & 10,0 \\
\hline spp & 1 & 1 & 0,7 & 0 & 0,0 & 1 & 2,3 & 0 & 0,0 \\
\hline Salmonella spp & 23 & 21 & 15,2 & 18 & 28,1 & 2 & 2,3 & 2 & 6,6 \\
\hline Serratia ॥ & 4 & 3 & 2,2 & 1 & 1,6 & 1 & 2,3 & 1 & 3,3 \\
\hline \multicolumn{10}{|l|}{ Aeromonaceae (10) } \\
\hline Aeromonas & 10 & 6 & 4,3 & 3 & 4,7 & 0 & 0,0 & 3 & 10,0 \\
\hline \multicolumn{10}{|l|}{ Moraxellaceae (11) } \\
\hline Acinetobacter & 11 & 5 & 2,8 & 0 & 0,0 & 5 & 11,4 & 0 & 0,0 \\
\hline \multicolumn{10}{|l|}{ Pseudomonaceae (8) } \\
\hline Pseudomonas & 8 & 7 & 5,1 & 3 & 4,7 & 2 & 4,5 & 2 & 6,6 \\
\hline
\end{tabular}

* Dentro de cada género se indican las especies detectadas en al menos un $10 \%$ de al menos un tipo de muestras o cuando todos los microorganismos restantes pertenecen a la misma especie

† Número de microorganismos, nótese que en todos los casos se reporta el total de cada tipo de microorganismos aislados de cada especie, pudiendo haber más de uno proveniente de la misma muestra

¥ Número de muestras en las que se aisló cada tipo de microorganismo, nótese que si en una muestra se aisló una misma especie de microorganismo varias veces la muestra sólo está considerada una vez

$\S$ Todos identificados como Morganella morganii

\| Todos identificados como Serratia marcescens

spp: especie perteneciente al género señalado 
spp. fue más frecuente en las muestras de pollo que en las muestras de carne de vacuno $(p<0,05)$; Citrobacter spp. fue más frecuentes en muestras de carne de vacuno que en muestras de pollo $(p<0,05)$ y Enterobacter spp. tendía a ser más frecuente en las muestras de cerdo que en las muestras de pollo.

\section{DETECCIÓN DE E. COLI DIARREOGÉNICAS}

Aunque se aislaron $407 \mathrm{E}$. coli, solo dos fueron clasificados como diarreogénicos $(0,5 \%$ de los aislados, $1,5 \%$ de las muestras), concretamente un STEC (presencia del gen stx) y un EPEC (presencia del gen eaeA) aislados de las muestras de cerdo $(3,3 \%)$ y de las muestras de res $(2,3 \%)$, respectivamente.

\section{NIVELES DE SENSIBILIDAD A ANTIMICROBIANOS}

Los niveles de sensibilidad a antimicrobianos se determinaron en 261 (64,1\%) de los aislados de E. coli (159 de pollo, 57 de vacuno y 45 de cerdo), que se obtuvieron de 56 muestras de pollo, 25 de vacuno y 22 de cerdo, respectivamente. A excepción de amoxicilina-ácido clavulánico $(p=0,337)$, el resto de los antimicrobianos presentaron diferencias significativas en los porcentajes de resistencia según el tipo de muestra $(p<0,05)$.

En los aislados de E. coli de muestras de pollo, el $100 \%$ fueron resistentes a cotrimoxazol, más del $90 \%$ fueron resistentes a ampicilina y tetraciclina, más del $80 \%$ fueron resistentes a quinolonas (ácido nalidíxico y ciprofloxacina) y cloranfenicol. En los antibióticos restantes, los niveles de resistencia fueron menores al $40 \%$, destacando la ausencia de resistencia a imipenem.

En los aislados de E. coli de muestras de cerdo, el $95 \%$ fueron resistentes a ampicilina, el $90 \%$ a tetraciclina, el $59,1 \%$ a ácido nalidíxico y el 54,5\% a cloranfenicol y cotrimoxazol. Además, un número considerable de aislados presentó resistencia intermedia a las quinolonas (ácido nalidíxico y ciprofloxacino).

En los aislados de E. coli de muestras de vacuno, el $52 \%$ fueron resistentes a tetraciclina, el $48 \%$ a ciprofloxacino y el $44 \%$ a ampicilina. Es importante destacar que el 98,2\% de $E$. coli de muestras de pollo fueron MDR. Los valores de $E$. coli MDR fueron menores en las muestras de cerdo (86,4\%), y mucho menores en las muestras de vacuno (28\%) (Tabla 2 ).

\section{PRESENCIA DE BLEE Y pAmpC}

Se analizaron 89 cepas de E. coli de 32 muestras de pollo y 62 cepas de $E$. coli de 20 muestras de carne de res. Los resultados mostraron la presencia de $E$. coli productor de BLEE en 19/32 (59,4\%) muestras de pollo, no se detectó la presencia de BLEE en las muestras de carne de vacuno. Mientras que $p A m p C$ sólo estuvo presente en tres muestras: $2 / 32(6,2 \%)$ en muestras de pollo y $1 / 20(5,0 \%)$ en muestras de vacuno.

\section{DISCUSIÓN}

En nuestro estudio, hemos encontrado una gran variedad de especies bacterianas, aunque no todos son patógenos, resalta la alta presencia de niveles de resistencia a los antibióticos. Un hallazgo preocupante es la presencia de Salmonella spp. en el $30 \%$ de las muestras de pollo. La Salmonella es un patógeno peligroso que comúnmente se asocia con diarrea, fiebre y calambres abdominales ${ }^{(3)}$, puede transmitirse directa o indirectamente entre animales y humanos, principalmente a través de alimentos contaminados ${ }^{(3)}$.

En general, el microorganismo detectado con mayor frecuencia fue E. coli, microorganismo cosmopolita y hospedero habitual de la microbiota intestinal en mamíferos y otros animales ${ }^{(17)}$. Es importante mencionar el bajo número de $E$. coli diarreogénicas encontradas $(0,5 \%)$, sobre todo porque reportes anteriores, que analizaron muestras humanas mostraron su presencia en el $43 \%$ de niños de áreas rurales de Perú ${ }^{(18)}$ y en el $30 \%$ de niños con o sin diarrea de áreas periurbanas de Lima ${ }^{(19)}$. Asimismo, su presencia en muestras de origen animal también ha sido reportada, incluyendo Lima y otras regiones peruanas ${ }^{(20,21)}$. La presencia de microorganismos resistentes a los antimicrobianos en los alimentos humanos es un problema creciente en América Latina ${ }^{(22)}$.

En nuestro estudio, observamos una variedad de aislados altamente resistentes a los antimicrobianos, con estimaciones de MDR de $80 \%$. Destaca la presencia de microorganismos productores de BLEE en muestras de pollo, y su no presencia en carne de vacuno. Aunque no se puede descartar la contaminación de E.coli productoras de BLEE en las muestras de pollo durante su manipulación en los mataderos o en el puesto de venta, esta amplia diferencia podría deberse primordialmente al uso de antibióticos en las granjas de pollos.

Aunque se sabe que el proceso de cocción del alimento eliminaría a las bacterias productoras de BLEE, se debe considerar la posible contaminación relacionada con la propia manipulación del producto, que facilitaría la propagación de estos microorganismos a las superficies, a alimentos no cocinados (por ejemplo, las ensaladas) y, posteriormente, al intestino humano ${ }^{(23)}$.

La mayoría de los microorganismos presentes, no son patógenos $\mathrm{y}$, por lo tanto, se pueden incorporar silenciosamente a la microbiota intestinal humana, ya sea de forma estable o inestable, actuando como portadores y/o reservorios de genes de resistencia a antimicrobianos. Estos podrían actuar como una especie de "caballo de Troya», pudiendo transferir estos genes a otros microorganismos residentes comensales o a microorganismos patógenos en infecciones que los hacen difíciles de tratar ${ }^{(24)}$. 
Tabla 2. Niveles de resistencia a los antibióticos en Escherichia coli aislados en carne de los mercados tradicionales de Lima

\begin{tabular}{|c|c|c|c|c|c|c|c|c|c|c|c|c|c|c|c|c|c|c|c|}
\hline \multirow{3}{*}{ Antibióticos } & \multicolumn{6}{|c|}{ Pollo (56) } & \multicolumn{6}{|c|}{ Vacuno (25) } & \multicolumn{6}{|c|}{ Cerdo (22) } & \multirow{3}{*}{$\begin{array}{l}\text { Valor } \\
\text { de } p^{\ddagger}\end{array}$} \\
\hline & \multicolumn{3}{|c|}{ Intermedia } & \multicolumn{3}{|c|}{ Resistente } & \multicolumn{3}{|c|}{ Intermedia } & \multicolumn{3}{|c|}{ Resistente } & \multicolumn{3}{|c|}{ Intermedia } & \multicolumn{3}{|c|}{ Resistente } & \\
\hline & $\mathbf{n}$ & $\%$ * & $\% \mathrm{O}^{\dagger}$ & $\mathbf{n}$ & $\%$ * & $\% \mathrm{O}^{\dagger}$ & $\mathbf{n}$ & $\%$ * & $\% \mathrm{O}^{\dagger}$ & $\mathbf{n}$ & $\%$ * & $\% \mathrm{O}^{\dagger}$ & $\mathbf{n}$ & $\%$ * & $\%$ * & $\mathbf{n}$ & $\%$ * & $\% \mathrm{O}^{\dagger}$ & \\
\hline Ampicilina & 0 & 0 & 0,0 & 53 & 94,6 & 90,1 & 0 & 0,0 & 0,0 & 11 & 44,0 & 31,0 & 0 & 0,0 & 0,0 & 21 & 95,4 & 76,0 & $<0,001$ \\
\hline $\begin{array}{l}\text { Amoxicilina } \\
\text { Ácido Clavulánico }\end{array}$ & 14 & 25,0 & 23,8 & 9 & 16,1 & 15,3 & 0 & 0,0 & 0,0 & 4 & 16,0 & 11,3 & 0 & 0,0 & 0,0 & 1 & 4,5 & 3,6 & 0,377 \\
\hline Azitromicina & 0 & 0,0 & 0,0 & 22 & 39,3 & 37,4 & 0 & 0,0 & 0,0 & 2 & 8,0 & 5,6 & 0 & 0,0 & 0,0 & 0 & 0,0 & 0,0 & 0,002 \\
\hline Cloranfenicol & 1 & 1,8 & 1,7 & 46 & 82,1 & 78,3 & 1 & 4,0 & 2,8 & 1 & 4,0 & 2,8 & 0 & 0,0 & 0,0 & 12 & 54,5 & 43,6 & $<0,001$ \\
\hline Ácido nalidixico & 3 & 5,4 & 5,1 & 50 & 89,3 & 85,1 & 7 & 28,0 & 19,7 & 4 & 16,0 & 11,3 & 4 & 18,2 & 14,5 & 13 & 59,1 & 47,3 & $<0,001$ \\
\hline Ciprofloxacino & 7 & 12,5 & 11,9 & 47 & 83,9 & 80,0 & 12 & 48,0 & 33,8 & 0 & 0,0 & 0,0 & 10 & 45,5 & 36,4 & 8 & 36,4 & 29,1 & $<0,001$ \\
\hline Furazolidona & 3 & 5,4 & 5,1 & 17 & 30,4 & 28,9 & 0 & 0,0 & 0,0 & 1 & 4,0 & 2,8 & 2 & 9,0 & 7,3 & 5 & 22,7 & 18,2 & 0,031 \\
\hline Cotimoxazol & 0 & 0,0 & 0,0 & 56 & 100,0 & 95,3 & 2 & 8,0 & 5,6 & 3 & 12,0 & 8,4 & 0 & 0,0 & 0,0 & 12 & 54,5 & 43,6 & $<0,001$ \\
\hline Tetraciclina & 0 & 0,0 & 0,0 & 53 & 94,6 & 90,1 & 1 & 4,0 & 2,8 & 13 & 52,0 & 36,6 & 0 & 0,0 & 0,0 & 20 & 90,9 & 72,7 & $<0,001$ \\
\hline MDR § & 55 & 98,2 & 93,6 & - & - & - & 7 & 28,0 & 19,7 & - & - & - & 19 & 86,4 & 69,1 & - & - & - & $<0,001$ \\
\hline PanS § & 0 & 0,0 & 0,0 & - & - & - & 4 & 16,0 & 11,3 & - & - & - & 1 & 4,5 & 3,6 & - & - & - & 0,008 \\
\hline
\end{tabular}

MDR: multidrogorresistente, Pans: pansensible

* porcentaje de muestras intermedias calculado sobre el número de muestras incluidas en presente análisis ( $\%=n \times 100 / n$ (muestra)

† porcentaje de muestras resistentes calculado sobre el total de muestras recolectadas ( $\% \mathrm{O}=\%$ x factor de corrección)

Los factores de corrección utilizados para calcular cada \%O son 0,953 (pollo), 0,704 (vacuno) y 0,80 (cerdo)

¥ Prueba de Chi Cuadrado o prueba de Fisher, según corresponda

$\S$ Se refiere al total de la muestra

No se encontró resistencia a Imipenem

Los altos niveles de resistencia podrían explicarse por el uso indiscriminado de antibióticos, para el tratamiento y profilaxis (aminoglucósidos, cefalosporinas, betalactámicos, quinolonas, macrólidos, tetraciclinas, fenicoles y sulfonamidas, principalmente) en animales de Perú ${ }^{(25)}$, cuyo riesgo puede ser aún mayor cuando estos antibióticos se usan por más tiempo, como comúnmente sucede en la producción de animales ${ }^{(26)}$. En Perú, los antibióticos más utilizados en veterinaria incluyen oxitetraciclina, penicilina y cotrimoxazol, según un estudio realizado en pequeñas granjas lecheras en las zonas rurales del norte del país ${ }^{(27)}$. En nuestro estudio, los niveles de resistencia más altos se encontraron para ampicilina, amoxicilina, cotrimoxazol y tetraciclina, todos ellos usados en veterinaria ${ }^{(25,27)}$. Algunos países de América Latina, incluido Perú, han implementado alternativas para controlar el uso de antibióticos, limitando el uso de cloranfenicol, olaquindox, nitroimidazoles y nitrofuranos en animales para consumo humano ${ }^{(28)}$.

Para promover el uso responsable de los antibióticos, se han publicado directrices internacionales y nacionales con el propósito de garantizar la eficacia terapéutica y mitigar la resistencia antimicrobiana ${ }^{(29)}$. En el presente estudio, los microorganismos de E. coli aislados de las muestras de pollo exhiben los mayores niveles de resistencia, este fenómeno ya ha sido descrito en otros estudios ${ }^{(30)}$. Este hallazgo podría estar relacionado con el proceso industrial en la producción de carne de pollo, de gran importancia en Perú, por el alto consumo de sus pobladores.

La Administración de Alimentos y Medicamentos (FDA, por sus siglas en inglés) propone diferentes estrategias para reducir y controlar el uso de antibióticos en veterinaria, como la promoción del veterinario con licencia, el control del manejo de la enfermedad y la prevención de antibióticos en animales productores de alimentos. Sin embargo, los resultados de nuestro estudio sugieren que estas medidas no serían suficientes. Los antibióticos probados en este estudio también se usan en humanos y la Organización Mundial de la Salud (OMS) declaró que la mayoría de estos antibióticos son clasificados como «críticamente importantes» o «muy importantes» para su uso como tratamiento en humanos ${ }^{(31)}$. Además, los mercados tradicionales también deben emplear buenas prácticas alimentarias e incluir el lavado y la desinfección, durante la preparación, el almacenamiento y el proceso de venta.

Como limitación del estudio se puede mencionar la selección del 64,1 \% (261/407) de aislados de E.coli para la realización del antibiograma, de estas sólo se consideraron 89 aislados para la búsqueda de BLEE y pAmpC. A pesar de esta limitación, se considera que la muestra analizada es representativa del total de aislados, por haberse realizado una selección aleatoria de los microorganismos incluidos.

En Perú, es necesario promover investigaciones adicionales en microorganismos resistentes a los antimicrobianos en la interfaz humano-animal-ambiente para mostrar las rutas de transmisión de bacterias resistentes, así como los genes de resistencia circulantes y el impacto de las presiones selectivas en varios reservorios (animales, humanos y el entorno). Este conocimiento permitirá mejorar las estrategias y programas locales dirigidos a reducir la carga de resistencia a los antibióticos, así como, contribuir a los esfuerzos colectivos para salvaguardar los antibióticos 
utilizados en humanos y luchar contra el creciente problema de salud pública que es la resistencia a los antibióticos.

En conclusión, este estudio presenta elevados niveles de resistencia a los antimicrobianos frecuentemente utilizados por humanos; además, se destaca que la carne de pollo y de vacuno pueden ser potenciales reservorios de $E$. coli productores de BLEE y $\mathrm{pAmpC}$, respectivamente.

Agradecimientos: Agradecemos a todos los vendedores de carne de los mercados tradicionales de Lima que amablemente colaboraron en este estudio. Además, a los miembros del laboratorio de Enfermedades Entéricas del Instituto de Medicina Tropical Alexander Von Humboldt.
Contribuciones de autoría: MJP, JR participaron en la concepción y diseño del artículo; LRR, SMM, CG, NP, MR, KO, DD, participaron en la recolección de resultados; MJP, JR participaron en análisis e interpretación de datos; MJP, JR, CG, TJO participaron en redacción del artículo; todos los autores realizaron la revisión crítica del artículo y aprobaron la versión final.

Fuentes de financiamiento: Este trabajo fue apoyado por la Sociedad Española de Enfermedades Infecciosas y Microbiología Clínica 2012 (Búsqueda de antibióticos y microorganismos resistentes en animales de consumo humano y piensos animales); JR fue apoyado por el programa I3 del Ministerio de Economía y Competitividad, España (número de concesión: CES11 / 012).

Conflictos de interés: Los autores no declaran conflictos de interés.

\section{REFERENCIAS BIBLIOGRÁFICAS}

1. Foley SL, Lynne AM. Food animal-associated Salmonella challenges: pathogenicity and antimicrobial resistance. J Anim Sci. 2008;86(14 Suppl):E173-87.

2. World Health Organization. WHO list of Critically Important Antimicrobials for Human Medicine (WHO CIA list). Geneva: WHO; 2015. Available in: http://who.int/foodsafety/publications/ cia2017.pdf

3. Baylis $\mathrm{C}$, Uyttendaele $\mathrm{M}$, Joosten $\mathrm{H}, \mathrm{Da}$ viesA. The Enterobacteriaceae and their significance to the food industry. Brussels: International Life Sciences Institute (ILSI); 2011. Disponible en: http://ilsi.eu/ wp-content/uploads/sites/3/2016/06/ EP-Enterobacteriaceae.pdf

4. Smith JL, Fratamico PM, Gunther NW 4th. Shiga toxin-producing Escherichia coli. Adv Appl Microbiol. 2014;86:14597. doi: 10.1016/B978-0-12-8002629.00003-2.

5. Pons MJ, Mosquito S, Gomes C, Del Valle LJ, Ochoa TJ, Ruiz J. Analysis of quinolone-resistance in commensal and diarrheagenic Escherichia coli isolates from infants in Lima, Peru. Trans R Soc Trop Med Hyg 2014;108:22-8.

6. Levy SB, FitzGerald GB, Macone AB. Spread of antibiotic-resistant plasmids from chicken to chicken and from chicken to man. Nature. 1976;260:40-2.

7. Palma N, Pons MJ, Gomes C, Mateu J, Riveros M, García W et al. Resistance to quinolones, cephalosporins and macrolides in Escherichia coli causing bacteraemia in Peruvian children. J Global Antimicrob Resist 2017;11: 28-33.

8. Rivera-Jacinto MA. Betalactamasas de espectro extendido en cepas de Escherichia coli y Klebsiella sp. aisladas de reservorios inanimados en un hospital del norte del Perú. Rev Esp Quimioter 2012; 25:161163.

9. Clinical and Laboratory Standards Institute. 2009. Performance standards for antimicrobial disk susceptibility tests; approved standard. Document M02-A10. tenth edition, Wayne, PA: CLSI.

10. International Organization for standardization (ISO) [Internet]. Geneva: ISO 21567:2004 Microbiology of food and animal feeding stuffs -- Horizontal method for the detection of Shigella spp. Available in: https://www.iso.org/standard/34612. html

11. Murray PR, Baron EJ, Jorgensen JH, Landry ML, Pfaller MA. Manual of Clinical Microbiology, 9th edition. Washington, Dc: ASM Press; 2007.

12. Barletta F, Mercado E, Lluque A, Ruiz J, Cleary T, Ochoa T. Multiplex real-time PCR for detection of Campylobacter, Salmonella, and Shigella. J Clinical Microb 2103; 51:2822-29.

13. Walk ST, Alm EW, Gordon DM, Ram JL, Toranzos GA, Tiedje JM, et al.. Cryptic lineages of the genus Escherichia. Appl Environ Microbiol 2009; 75:6534-44.

14. Salazar de Vegas EZ, Nieves B, Araque M, Velazco E, RuizJ, Vila J. Outbreak of infection with Acinetobacter strain RUH 1139 in an Intensive Care Unit. Infect Control Hosp Epidemiol 2006; 27:397-403.

15. Guion CE, Ochoa TJ, Walker CM, Barletta F, Cleary TG. Detection of diarrheagenic Escherichia coli by use of melting-curve analysis and real-time multiplex PCR. J Clin Microbiol 2008; 46:1752-7.
16. Pons MJ, Gomes C, Martínez-Puchol S, Ruiz L, Mensa L, Vila J, et al. Antimicrobial resistance in Shigella spp. causing traveller's diarrhoea (1995-2010): a retrospective analysis. Travel Med Infect Dis 2013; 11,315-19

17. Eguale T, Gebreyes W, Asrat D, Alemayehu H, Gunn JS, Engidawork E. Non-typhoidal Salmonella serotypes, antimicrobial resistance and co-infection with parasites among patients with diarrhea and other gastrointestinal complaints in Addis Ababa, Ethiopia. BMC Infect Dis 2015;15:497.

18. Acosta GJ, Vigo NI, Durand D, Riveros M, Arango S, Zambruni M, et al. Diarrheagenic Escherichia coli: Prevalence and Pathotype Distribution in Children from Peruvian Rural Communities. Am J Trop Med Hyg 2016; 7:574-9.

19. Ochoa TJ, Ruiz J, Molina M, Del Valle LJ, Vargas M, Gil et al. High frequency of antimicrobial drug resistance of diarrheagenic Escherichia coli in infants in Peru. Am J Trop Med Hyg 2009;81:296-301.

20. Caballero M, Rivera I, Jara LM, Ulloa-Stanojlovic FM, Shiva C. I Isolation and molecular identification of potentially pathogenic Escherichia coli and Campylobacter jejuni in feral pigeons from an urban area in the city of Lima, Peru. Rev Inst Med Trop Sao Paulo 2015; 57:393-6.

21. Rivera FP, Sotelo E, Morales I, Menacho F, Medina AM, Evaristo R, et al. Short communication: Detection of Shiga toxin-producing Escherichia coli (STEC) in healthy cattle and pigs in Lima, Peru. J Dairy Sci 2012:95:1166-9. 
22. Gómez-Aldapa CA, Segovia-Cruz JA, Cerna-Cortes JF, Rangel-Vargas E, Salas-Rangel LP, Gutiérrez-Alcántara EJ, et al. Prevalence and behavior of multidrug-resistant shiga toxin-producing Escherichia coli, enteropathogenic $E$. coli and enterotoxigenic $E$. coli on coriander. Food Microbiol 2016:59:97-103.

23. Leverstein-van Hall MA, Dierikx CM, Cohen Stuart J, Voets GM, van den Munckhof MP, van Essen-Zandbergen A, et al. Dutch patients, retail chicken meat and poultry share the same ESBL genes, plasmids and strains. Clin $\mathrm{Mi}$ crobiol Infect 2011;17(6):873-80.

24. Bidet P, Burghoffer B, Gautier V, Brahimi N, Mariani-Kurkdjian P, ElGhoneimi A et al. In vivo transfer of plasmid-encoded ACC-1 AmpC from Klebsiella pneumoniae to Escherichia coli in an infant and selection of impermeability to imipenem in K. pneumoniae. Antimicrob Agents Chemother 2005; 49:3562-3565.

25. Ministerio de Salud. Norma sanitaria que establece los límites máximos de residuos (lmr) de medicamentos veterinarios en alimentos de consumo humano [Internet]. Lima: MINSA; 2012. Disponible en: ftp://ftp2.minsa.gob.pe/normaslegales/2012/RM739_2012_MINSA.pdf

26. De Briyne N, Atkinson J, Borriello SP, Pokludová L. Antibiotics used most commonly to treat animals in Europe. Veterinary Record 2014; 175: 325.

27. Redding LE, Cubas-Delgado F, Sammel MD, Smith G, Galligan DT, Levy MZ, et al. The use of antibiotics on small dairy farms in rural Peru. Vet Med 2014;113;88-95.

28. Quesada A, Reginatto GA, Ruiz Español A, Colantonio LD, Burrone MS. Resistencia antimicrobiana de Salmonella spp. aislada de alimentos de origen animal para consumo humano. Rev Peru Med Exp Salud Publica. 2016;33:32-44.

29. Bentsson B, Greko C. Antibiotic resistance-consequences for animal health, welfare, and food production. Ups J Med Sci 2014;119:96-102.

30. Messele YE, Abdi RD, Yalew ST, Tegegne DT, Emeru BA, Werid GM. Mo- lecular determination of antimicrobial resistance in Escherichia coli isolated from raw meat in Addis Ababa and Bishoftu, Ethiopia. Ann Clin Microbiol Antimicrob 2017;16:55.

31. World Health Organization, World Health Day: Food Safety [Internet]. Genova: World Health Organization Publishing; 2015. Available in: http:// www.who.int/campaigns/worldhealth-day/2015/event/en/

Correspondencia: Maria J. Pons, PhD

Dirección: Laboratorio de Microbiología Moleculary Genómica Bacteriana. Universidad Cientifica del Sur, Antigua Panamericana Sur, Km19 Lima, Peru.

Teléfono: (+51)930491968

Correo electrónico:ma.pons.cas@gmail.com

\section{Ahora nuestra revista incluye:}

Publicación anticipada

$\checkmark$ Compartiendo publicaciones científicas con el ciudadano

$\checkmark$ Videos de presentaciones conjuntas del Instituto Nacional de

Salud y la Academia Nacional de Medicina

$\checkmark$ Galería fotográfica y uideos

https://rpmesp.ins.gob.pe 